\title{
Cell-cell fusion as a potential target in cancer therapy
}

\author{
JM Gasent Blesa ${ }^{1}$ and VA Candel ${ }^{2}$ \\ ${ }^{1}$ Hospital General Universitari Marina Alta, Denia, Plana de l'Est no 5, Alacant, Spain \\ ${ }^{2}$ Hospital Universitari Arnau de Vilanova, Valencia, Spain
}

\begin{abstract}
In the fight against cancer, new and more specific targets are needed. Here, we offer an example of a potential target that has not been widely studied, namely the syncytin protein. Syncytin is expressed mainly in the human placenta and is implicated in placental syncytiotrophoblast cell fusion. Not much is known about the role of syncytin in cancer, but the existing data call for more intense research. Its retroviral origin and particular tissue distribution make syncytin an interesting potential target in cancer therapy.
\end{abstract}

Published: 06/08/2009

Received: $17 / 05 / 2009$

ecancer 2009, 3:145 DOI: 10.3332/ecancer.2009.145

Copyright: (c) the authors; licensee ecancermedicalscience. This is an Open Access article distributed under the terms of the Creative Commons Attribution License (http://creativecommons.org/licenses/by/2.0), which permits unrestricted use, distribution, and reproduction in any medium, provided the original work is properly cited.

Competing Interests: The authors have declared that no competing interests exist.

Correspondence to JM Gasent Blesa. Email: joanmagasent@telefonica.net 


\section{Translational relevance}

This paper reviews an unexplored potential new target in cancer therapy. We consider the origin of syncytin, its normal role in human placentogenesis and data regarding its relevance in cell-cell fusion. Given its particular distribution in normal human tissues and its potential roll as an immune modulator and in amino acid transport, tissue tolerance and protection against retroviral infection, syncytin could be a potential target for immune therapy using specific inhibitors. Current data about the role of syncytin in cancer are also discussed.

\section{Introduction}

Transposable elements (TEs) or mobile elements are genetic components that can have a significant effect on the host genome [1,2]. Furthermore, these elements form the majority of the human genome. Insertions of TEs influence the transcriptional regulation of some genes as well as the termination of transcription. Hundreds of examples have been identified in which individual exons possess sequences that are similar or identical to TE fragments $[3,4]$. In many of these cases, a single exon is involved, and its transcription yields a variant mRNA [5]. It has been suggested that TEs are a source of variation as a result of the insertion of fragments of their sequence into functioning genes elsewhere in the genome.

One of the potential effects of TEs is the generation of new gene sequences $[3,6,7]$ such as the envelope (env) genes of retroviral origin found in several mammals. In particular, the human genome contains full-length copies of four env openreading frames (ORFs) that are highly expressed in the placenta [8-10].

The best known of the human env-derived genes is syncytin-1, or ERVWE1, which originated from a human endogenous retrovirus (HERV) of the HERV-W family inserted on chromosome 7q21 in human-pre-hominoids 40-45 million years ago $[11,10]$. The env gene maintained an ORF coding for a 538amino acid polypeptide that has all the characteristic features of env proteins and mediates intercellular fusion in vitro [11-13].

Recently, a molecular evolution study of the HERV-W provirus in several ape species and 24 humans demonstrated the conservation of syncytin-1, as well as its retention of receptormediated fusogenic activity [14]. However, an analysis of the synonymous and non-synonymous substitutions indicated a relatively high degree of amino acid change in hominoids [15], which could be consistent with a low degree of selective pressure. HERV-W invasion of the primate genome has been documented as occurring before the divergence of hominoids and Old World monkeys [16,17] and as being inactive [18].

Another syncytin, syncytin-2, has also been identified; it is functional in all primates, including New World monkeys, but it is absent from prosimians, thus dating its entry into the primate genome to about 40 million years ago $[19,20]$. The syncytin-2 gene encodes a $529-\mathrm{kDa}$ polypeptide with a general organization similar to that of retroviral envelopes: a cleavage site separates the surface subunit that binds a still unidentified receptor on the target cell from the transmembrane subunit (TM), which is classically thought to be involved in the fusion process [21]. The TM subunit harbours an $\mathrm{N}$-terminal fusion peptide, an ectodomain with a leucine zipper motif, a highly hydrophobic transmembrane anchor and a C-terminal intracellular domain.

The ectodomains of retroviral envelopes are believed to play a pivotal role in their fusogenic function. Indeed, by analogy with the pre-fusion and post-fusion structures of influenza haemagglutinin, they include a short loop that undergoes a dramatic change in geometry during the fusion process [22,23] switching the ectodomain from an entirely a-helical to a helixturn-helix conformation. When the structure of the syncytin-2 central domain is compared to that of present-day retroviruses, such as MoMLV and the human T-cell lymphotropic virus (HTLV-I), the geometries of the pivotal loops of all three ectodomains appear virtually identical, despite completely different amino acid sequences [24].

\section{Functions of syncytin}

The HERV-derived syncytin may have played a role in the evolution of the human placenta due to its niche function in human placentogenesis, albeit in a highly specific manner, as the placentas of different mammalian species exhibit structural discrepancies [25].

Because of its interaction with a specific receptor known to function as a retrovirus receptor and an amino acid transporter, and its stimulation of cell-cell fusion processes, the protein was designated as syncytin (Figure 1).

The syncytin receptor, or at least the first known functional syncytin-binding site, is the sodium-dependent neutral amino 


\section{Syncytin as a Target and its Functions}

TARGETING SYNCYTIN

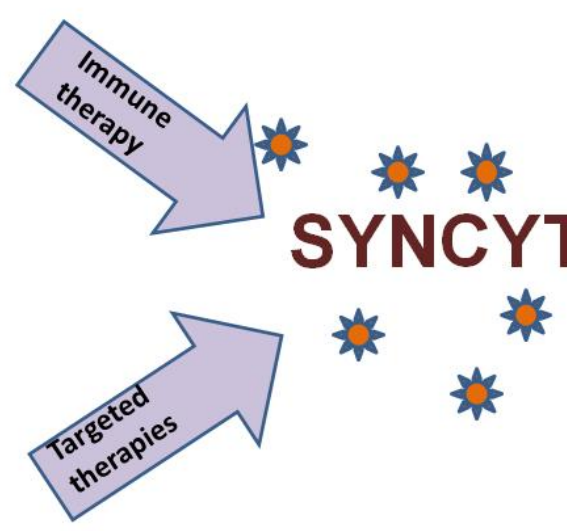

SYNCYTIN FUNCTIONS

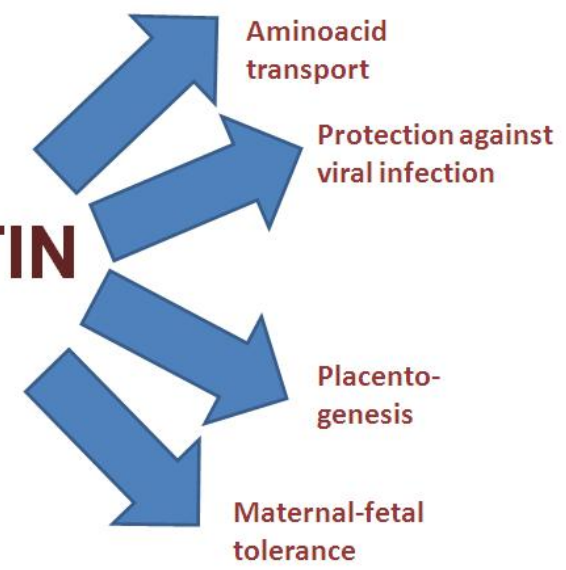

Figure 1: Potential functions of syncytin—targeting syncitin.

acid transporter ASCT2, which transports alanine, serine and cysteine. Elevated syncytin expression is followed by further cell differentiation and generation of the syncytium, formation of gap junctions and an increase in $\beta$-hCG secretion [26]. The effects of syncytin can be blocked in vitro by antibodies directed against syncytin or by the use of syncytin antisense strategies $[11,26]$. In addition to in vitro studies, it was recently demonstrated that the syncytin locus is strongly preserved in a large cohort of individuals, including long-terminal repeat (LTR) elements involved in the regulation of gene transcription [14]. Apart from these findings, orthologous syncytin loci have been identified in the genomes of great apes [14]; moreover, expression of syncytin has been described in the rhesus monkey endometrium, where it may play a role in decasualization or receptivity [27].

ASCT2 expression has been demonstrated predominantly at the basal membrane of the human syncytiotrophoblast [28-31], which may function as the interaction site between syncytin and its receptor. The observation that syncytin and ASCT2 levels are inversely correlated during cell syncytialization could be partially explained by the phenomenon that retroviruses may induce down-regulation of their receptors [32]. This can be observed after infection of cells with wild-type viruses of the type-D interference group, which impairs neutral amino acid transport [33].

It has been previously reported that an alteration in particular placental amino acid transporters is associated with intrauterine growth restriction (IUGR) and distinctive anthropometric features in the foetus, especially for system $A$, which transports neutral amino acids [34-36].

System A, in contrast to the ASCT2 transporter, is downregulated in the case of oxygen deficiency and up-regulated in the case of excess oxygen [28], which may contribute to foetal IUGR. Other transport systems, for example those for arginine, have been mentioned in the context of pre-eclamptic placentas [37].

Another important point concerning syncytin is its potential role in maternal immune tolerance to the foetus. Exogenous retroviral envelope proteins can inhibit the immune responses of leukocytes by means of a highly consistent amino acid sequence [38]. This sequence in the syncytin protein may therefore contribute to a specific immune modulating mechanism within the developing placenta [11]. The nature of 
additional interactions with other mechanisms such as the $\mathrm{MHC}$ class I molecules, in particular, HLA-G, which is thought to produce immune tolerance [39], or immunoregulatory cytokines such as immunosuppressive interleukin-10 [40] remains unclear, although syncytiotrophoblast formation stimulated by syncytin may subsequently ensure syncytiotrophoblast survival via additional interactions with immune or apoptotic mechanisms. Whether, or to what extent, syncytin contributes to maternal immune tolerance of the foetus or interacts with other mechanisms such as apoptotic events at the maternal-foetal interface is currently unclear.

Syncytin expression has been shown to cause cellular resistance to infection by spleen necrosis virus [41], and it is likely to prevent infection by other retroviruses of the same family. It is thus feasible that expression of syncytin by the syncytiotrophoblast prevents vertical transmission of a number of retroviruses through the placenta.

\section{Gene promoter: description, mutations and repercussions. Fusion in cell lines}

The major transcription initiation site of syncytin-1 gene is 56 base pairs (bp) downstream from a putative CCAAT box [42]. Deletion analysis performed on the 59-bp flanking region of the syncytin gene indicated that the proximal $148 \mathrm{bp}$ are essential for minimal promoter activity and that specific regions of the promoter from nucleotides (nts) -1519 to -984 and nts -294 to -148 are required for maximal expression in normal trophoblast cells. DNAse-I footprint analysis of the region between nts -252 and +110 revealed three protected regions, FP1-FP3. Mutagenesis of a hepatocyte-specific nuclear protein-1-binding site in FP1 and a TATA box in FP3 had no effect on basal promoter activity. On the other hand, mutation of the CCAAT motif and the octamer protein binding site in FP2 decreased promoter activity by $88 \%$ and $76 \%$, respectively. Mutation of the ecdysone receptor response element in FP2, which may bind a nuclear hormone receptor, increased basal promoter activity. Gel shift and supershift assays indicated that the CCAATbinding factor binds to the CCAAT motif and that octamer binds to the octamer-binding site. Thus, it can been deduced that the syncytin promoter is located in the 59-bp-long terminal repeat of the HERV-W gene and that binding sites for GATA and Oct in the proximal promoter are critical for transcriptional regulation of syncytin in trophoblast cells [42].

The 146-bp stretch of the 59-bp flanking region in the human syncytin gene from nts 294 to 148 is essential for basal gene expression in human BeWo and JEG3 choriocarcinoma cell lines, but not in hepatoblastoma and kidney cell lines. Ligation of the 146-bp fragment to an SV40 (Simian Virus 40) promoter or a human $\beta$-globin minimal promoter markedly enhanced promoter activity in placental cells, but not in liver or kidney cells. DNase-I footprint assays indicated that nuclear extracts from BeWo cells, but not HepG2 cells, protected four regions (FP1-FP4) of the 146-bp fragment. Furthermore, site-directed mutagenesis of an SP1-binding site in FP3 and a GATA-binding site in FP4 significantly repressed promoter activity in the placental cells. Over-expression of SP1 (Sp1 transcription factor), GATA2 (GATA binding protein 2) and GATA3 induced syncytin promoter activity but had little or no effect on the activity of syncytin promoter fragments containing mutations in the SP1- and GATA-binding sites. In addition, GATA2 and GATA3 mRNA levels markedly increased during spontaneous in vitro differentiation of human cytotrophoblast cells after they fused to form a syncytium. These findings suggest that the 146bp region of the 59-bp flanking region (nts 2294/2148) of the human syncytin gene acts as a placenta-specific enhancer. Furthermore, binding of SP1 and GATA family members to this enhancer is critical for cell-specific expression of the syncytin gene [43].

\section{Fusion in cancer cells}

Mortensen et al [44] found that human breast cancer cells fused with endothelial cells in culture. Expression of syncytin-1 protein has also been observed in breast cancer cell lines [45], and both tumour cells and endothelial cells express the syncytin-1 receptor ASCT-2. Using syncytin-1 antisense oligonucleotides to down-regulate syncytin-1 expression inhibited breast cancerendothelial cell fusion. In addition, the syncytin-1 CHR peptide also inhibited cell fusion [45]. Neither the antisense nor the CHR peptide experiments, however, effected total inhibition of cancer-endothelial cell fusion. It is possible that the antisense oligonucleotides did not completely eliminate syncytin-1 expression. On the other hand, syncytin-2 protein expression has also been documented and could contribute to cancer-host cell fusion. Further experiments using shRNA-directed downregulation of both syncytin-1 and -2 proteins, as well as additional putative fusogenic retroviral sequences, are underway to test this possibility [46].

These data provide strong evidence that syncytin-1 protein is involved in mediating cancer-endothelial cell fusion in vitro.

The involvement of syncytin-1 in tumour cell fusion events was subsequently confirmed by Strick et al [47] in endometrial carcinomas. In their study, down-regulation of synctytin-1 
protein expression also inhibited fusion between endometrial tumour cells. In agreement with findings in placental cells, both CAMP-elevating agents and oestrogens up-regulated syncytin-1 protein expression; however, only cAMP-elevating agents stimulated cell fusion [48]. This apparent discrepancy could be ascribed to the fact that oestrogen treatment up-regulates TGF beta, which interferes with syncytin-induced fusion. Indeed, oestrogen did stimulate cell fusion if TGF beta was neutralized with antibodies. Conversely, addition of TGF beta 1 or 3 reduced the cell fusion induced by cAMP-elevating agents. This effect was observed in both endometrial carcinoma cells and trophoblast-derived cells. These results show that both CAMP and oestrogens may positively regulate syncytin-1 protein expression in tumour cells and that TGF beta family members may negatively regulate the fusogenic effects of syncytin- 1 in both trophoblasts and cancer cells [48]. The role of syncytin-1 and cell fusion in cancer requires further study.

Published data show that the syncytin-1 protein is not the only fusogenic protein expressed by breast cancer cells, and the results presented by Strick et al [47] show that additional regulators, such as TGF beta isoforms, may be important modulators of cell fusion.

This is not to say that cell fusion may be universally beneficial to all cancers. First, syncytins may have additional effects within the tumour environment $[46,47]$. Second, it seems likely that several factors within the cancer and its stroma (e.g., inactivated tumour suppressor genes, activated oncogenes and expression of fusogens and of CD9, CD81 and TGF beta) may function together to bring about a tumour profile as diverse as that demonstrated by the cell fusion experiments referenced above. Expression of CD9 has been analysed in a number of tumours and seems, like syncytin expression, to correlate with a good prognosis [48-50].

\section{Syncytin and endometrial carcinoma}

The vast majority of endometrial carcinomas express the oestrogen and progesterone receptors and are thought to develop from endometrial hyperplasia induced by steroid hormone stimulation. Strick et al [47] demonstrated that syncytin-1 expression is regulated via an oestrogen response element in these steroid-driven tumours, resulting in increased proliferation of primary endometrial carcinoma cells and cell lines. It is noteworthy that activation of cAMP signalling by forskolin or SP-cAMP also resulted in syncytin-1 gene upregulation, although this intervention led to increased cell-cell fusion instead of proliferation. Furthermore, they showed that, after stimulation with steroids or CAMP activation, syncytin-1 gene is involved in anchorage-independent colony growth and fusion. The switch between cell proliferation and cell--cell fusion was shown to be subject to a complex regulatory mechanism involving both steroid hormone- and TGF- $\beta 1 /$ TGF- $\beta 3$-dependent effects. The authors' findings raise the possibility that syncytins play an important role in the progression of hormone-dependent cancers, such as endometrial carcinoma. In healthy individuals, syncytin-1 protein expression is usually restricted to the placenta, and it has been suggested that $\mathrm{CpG}$ hypomethylation of the 5' LTRs of both syncytin genes is one of the underlying regulatory mechanisms [51]. CpG hypomethylation also appears to be a means of activating syncytin-1 protein expression in ovarian carcinoma [52].

Since syncytin is up=regulated in endometrial carcinoma, the results of Strick et al [47] suggest that syncytin-1-mediated cell fusion may promote rather than suppress tumour growth. They demonstrated that syncytin- 1 expression and function are highly dependent on hormonal regulation: steroid hormones such as estradiol (E2) induce the expression of syncytin-1 protein and result in increased cell proliferation and anchorage-independent growth. On the other hand, activation of cAMP signalling promotes both endometrial carcinoma cell fusion and anchorage-independent growth. Cell fusion can be blocked by TGF- $\beta 1 / 3$, which is itself subject to steroid hormone regulation. Thus, in the presence of steroid hormones, TGF- $\beta$ provides a switch between syncytin-1-mediated cell fusion and cell proliferation. Oestrogen is a key aetiological factor in endometrial carcinogenesis. Regulation by TGF- $\beta$ and the involvement of syncytin-1 add a new dimension to our understanding of endometrial carcinoma progression. These findings reveal potential new directions for therapeutic approaches that circumvent the need for potentially risky endocrinological interventions.

The identification of syncytin as a target of TGF- $\beta$ in endometrial carcinoma not only provides novel insight into the molecular mechanisms of tumour progression but may also have important clinical implications. A peptide capable of inhibiting syncytin-mediated cell-cell fusion was recently shown to reduce breast carcinoma cell fusion with human umbilical vein endothelial cells by $50 \%(p<0.001)$ [45]. While this inhibitor has been tested only in an experimental setting and its toxicity profile has not yet been evaluated, the use of this peptide or of related reagents may have beneficial effects in the treatment of endometrial and, possibly, other carcinomas. However, there is a need to evaluate the contribution of cell fusion to the malignant phenotype of endometrial carcinoma cells to rule out the possibility that expression of syncytin is a mere 
physiological response of the host to the tumour. Such a response could, for example, be aimed at neutralizing malignant cells via fusion with normal cells expressing tumour suppressor genes [53].

\section{Syncytin and breast cancer}

Two series of human breast cancer patients were evaluated for tumoural expression of syncytin-1, using a polyclonal antiserum raised against a synthetic nanopeptide derived from the syncytin-1 sequence. In addition, tumours were also screened for expression of ASCT-2 using a peptide antiserum. Preabsorption of the antisera with the corresponding peptides, but not with irrelevant peptides, abolished staining [54].

The results show that $38 \%$ of all breast cancer samples displayed detectable staining for syncytin and that endothelial cells expressed ASCT-2. Moreover, significant expression of ASCT-2 was detected in many tumour cells. The degree of syncytin immunostaining was visually graded using coded specimens and statistical analysis showed that it correlated positively with the disease-free survival of patients [47]. Multivariate analysis included age dichotomised at 40 years, tumour size dichotomised at $20 \mathrm{~mm}$, grade and adjuvant therapy, and it identified syncytin expression as an independent prognostic indicator of increased disease-free survival. When used as a continuous variable, syncytin expression emerged as a significant prognostic indicator of disease-free survival in the Cox model $(\mathbf{p}=0.02)[47]$.

\section{Conclusions}

We believe that syncytins may be important fusogens for both trophoblast and cancer cell fusion, and that they may mediate additional cell fusion events, acting in concert with other molecules that have both enhancing and inhibitory regulatory effects. Both effects are relevant in mammalian placentogenesis. Syncytins have also been implicated in amino acid transport [28], immune tolerance [38] and protection against retroviral infections [41]. These functions of syncytin could all be utilized by cancers to gain a growth advantage, and, in our opinion, these functions should be studied in neoplasias.

Correlations of syncytin with clinical parameters are scarce, but the most relevant data have been reported in breast cancer [53]. Besides laboratory approaches and clinical studies correlating syncytin-1 expression in endometrial carcinoma, breast carcinoma and other tumours, we believe that syncytin should be studied in relation to histopathological and clinicopathological parameters in order to assess the relevance of this potential novel molecular target. Its unique localization and distribution make syncytin attractive for the development of targeted therapies and immune therapies. 


\section{References}

1. Kidwell MG and Lisch D (1997) Transposable elements as sources of variation in animals and plants PrOC Natl Acad Sci USA 94 7704-11 PMID: 9223252 doi: 10.1073/ pnas.94.15.7704

2. McDonald JF (1993) Evolution and consequences of transposable elements Curr Opin Genet Dev 3 855-64 PMID: 8118210 doi: $10.1016 / 0959-437 X(93) 90005-A$

3. Nekrutenko A and Li WH (2001) Transposable elements are found in a large number of human protein-coding genes Trends Genet 17 619-21 PMID: 11672845 doi: 10.1016/S0168-9525(01)02445-3

4. Lorenc A and Makalowski W (2003) Transposable elements and vertebrate protein diversity Genetica 118 183-91 PMID: 12868608 doi: 10.1023/A:1024105726123

5. Sorek RR, Ast G and Graur D (2002) Alu-containing exons are alternatively spliced Genome Res 12 1060-7 PMID: 12097342 doi: $10.1101 / \mathrm{gr} .229302$

6. Britten RJ (2004) Coding sequences of functioning human genes derived entirely from mobile element sequences Proc Natl Acad Sci USA 101 16825-30 PMID 15546984 10.1073/pnas.0406985101

7. Smit AF (1999) Interspersed repeats and other mementos of transposable elements in mammalian genomes Curr Opin Genet Dev 9 657-63 PMID: 10607616 doi: 10.1016/S0959-437X(99)00031-3

8. Cohen M, Powers M, O'Connell C, and Kato N (1985) The nucleotide sequence of the env gene from the human provirus ERV3 and isolation and characterization of an ERV3-specific cDNA Virology 147 449-58 PMID: 3840930 doi: $10.1016 / 0042-6822(85) 90147-3$

9. Blaise S, de Parseval $N$ and Heidmann $T$ (2005) Functional characterization of two newly identified human endogenous retrovirus coding envelope genes Retrovirology 219 PMID: 15766379 doi: 10.1186/17424690-2-19

10. Blond JL, Beseme F and Duret L (1999) Molecular characterization and placental expression of HERV-W, a new human endogenous retrovirus family $\mathrm{J}$ Virol 73 1175-85 PMID: 9882319

11. Mi S, Lee X, Li X et al (2000) Syncytin is a captive retroviral envelope protein involved in human placental morphogenesis Nature 403 785-9 PMID: 10693809 doi: $10.1038 / 35001608$

12. Blond JL, Lavillette $D$, Cheynet $V$ et al (2000) An envelope glycoprotein of the human endogenous retrovirus HERV-W is expressed in the human placenta and fuses cells expressing the type $D$ mammalian retrovirus receptor J Virol 74 3321-9 PMID: 10708449 doi: 10.1128/ JVI.74.7.3321-3329.2000

13. Frendo JL, Olivier D and Cheynet V (2003) Direct involvement of HERV-W Env glycoprotein in human trophoblast cell fusion and differentiation $\mathrm{Mol}$ Cell Biol 23 3566-74 PMID: 12724415 doi: 10.1128/MCB.23.10. 3566-3574.2003

14. Mallet F, Bouton O, Prudhomme S et al (2004) The endogenous retroviral locus ERVWE1 is a bona fide gene involved in hominoid placental physiology Proc Natl Acad Sci USA 101 1731-6 PMID: 14757826 doi: 10.1073/pnas.0305763101

15. Bonnaud B, Bouton O, Oriol G et al (2004) Evidence of selection on the domesticated ERVWE1 env retroviral element involved in placentation $\mathrm{Mol} \mathrm{Biol}$ Evol 21 1895901 PMID: 15254254 doi: $10.1093 / \mathrm{molbev} / \mathrm{msh} 206$

16. Voisset C, Blancher A, Perron $\mathrm{H}$ et al (1999) Phylogeny of a novel family of human endogenous retrovirus sequences, HERV-W, in humans and other primates AIDS Res Hum Retroviruses 15 1529-33 PMID: 10580403 doi: 10.1089/088922299309810

17. Kim HS, Takenaka $O$ and Crow TJ (1999) Isolation and phylogeny of endogenous retrovirus sequences belonging to the HERV-W family in primates $\mathrm{J}$ Gen Virol 80 2613-9 PMID: 10573154

18. Caceres M (2006) Thomas The Gene of Retroviral Origin Syncytin 1 is Specific to Hominoids and is Inactive in Old World Monkeys J Heredity 97 100-6 PMID: 16424151 doi: 10.1093/ihered/esj011

19. Blaise S, de Parseval N, Bénit L and Heidmann T (2003) Genome-wide screening for fusogenic human endogenous retrovirus envelopes identifies syncytin 2 , a gene conserved on primate evolution Proc Natl Acad Sci USA 100 13013-8 PMID 14557543 10.1073/pnas. 2132646100

20. Blaise S, Ruggieri A, Dewannieux M, Cosset $F-L$ and Heidmann T (2004) Identification of an envelope protein from the FRD family of Human Endogenous Retroviruses (HERV-FRD) conferring infectivity and functional conservation among simians J Virol 781050 4 PMID: 14694139 doi: 10.1128/JVI.78.2.1050-1054.2004

21. Colman PM and Lawrence MC (2003) The structural biology of type I viral membrane fusion Nat Rev Mol Cell Biol 4 309-19 PMID 12671653 10.1038/nrm1076

22. Bullough PA, Hughson FM, Skehel JJ and Wildley DC (1994) Structure of influenza hemagglutinin at the $\mathrm{pH}$ of membrane fusion Nature 371 37-43 PMID: 8072525 doi: 10.1038/371037a0

23. Chen J, Skehel JJ and Wiley DC (1999) N- and C-terminal residues combine in the fusion-Ph influenza 
hemagglutinin HA2 subunit to form an N CAP that terminates the triple-stranded coiled coil Proc Natl Acad Sci USA 96 8967-73 PMID: 10430879 doi: 10.1073/pnas. 96.16 .8967

24. Renard M, Varela PF, Letzelterl C, Duquerroy D, Rey F and Heidmann $T$ (2005) Crystal structure of a pivotal domain of human syncytin-2, a 40 million years old endogenous retrovirus fusogenic envelope gene captured by primates $\mathrm{J} \mathrm{Mol} \mathrm{Biol} 352$ 1029-34 PMID: 16140326 doi: $10.1016 / j . j m b .2005 .07 .058$

25. Stoye JP and Coffn JM (2000) A provirus put to work Nature 403 715-7 PMID: 10693785 doi: 10.1038/ $\underline{35001700}$

26. Frendo J-L, Olivier D, Cheynet V et al (2003) Direct involvement of HERV-W Env glycoprotein in human trophoblast cell fusion and differentiation $\mathrm{Mol} \mathrm{Cell} \mathrm{Biol}$ 23 3566-74 PMID: 12724415 doi: 10.1128/MCB.23.10. 3566-3574.2003

27. Okulitz WC and Ace Cl (2003) Temporal regulation of gene expression during the expected window of receptivity in the rhesus monkey endometrium Biol Reprod 69 1593-9 PMID: 12855598 doi: 10.1095/ biolreprod.103.017525

28. Nelson DM, Smith SD, Furesz TC, Sadovsky Y, Ganapathy V, Parvin CA and Smith $\mathrm{CH}$ (2003) Hypoxia reduces expression and function of system $A$ amino acid transporters in cultured term human trophoblasts $A m \mathrm{~J}$ Physiol Cell Physiol 284 C310-5 PMID: 12388062

29. Moe AJ (1995) Placental amino acid transport $A m \mathrm{~J}$ Physiol 268 C1321-31 PMID: 7611349

30. Kudo $Y$ and Boyd $C$ (2002) Changes in expression and function of syncytin and its receptor, amino acid transport system B (0) (ASCT2), in human placental choriocarcinoma BeWo cells during syncytialization Placenta 23 536-41 PMID: 12175968 doi: 10.1053/plac. 2002.0839

31. Furesz C, Smith CH and Moe AJ (1993) ASC system activity is altered by development of cell polarity in trophoblast from human placenta $A m$ J Physiol 265 C212-7 PMID: 8338132

32. Tailor CS, Nouri A, Zhao Y, Takeuchi Y and Kabat D (1999) A sodium-dependent neutral-amino-acid transporter mediates infections of feline and baboon endogenous $r$ etroviruses and simian type $D$ retroviruses $\mathrm{J}$ Virol 73 4470-4 PMID: 10196349

33. Rasko JE, Battini JL, Gottschalk RJ, Mazo I and Miller AD (1999) The RD114/simian type D retrovirus receptor is a neutral amino acid transporter Proc Natl Acad Sci USA 96 2129-34 PMID: 10051606 doi: 10.1073/pnas.96.5.2129
34. Norberg S, Powell TL and Jansson T (1998) Intrauterine growth restriction is associated with a reduced activity of placental taurine transporters Pediatr Res 44 233-8 PMID: 9702920 doi: 10.1203/00006450-199808000-00016

35. Glazier J, Cetin I, Perugino G, Ronzoni S, Grey AM, Mahendran D, Marconi AM, Pardi G and Sibley C (1997) Association between the activity of the system A amino acid transporter in the microvillous plasma membrane of the human placenta and severity of fetal compromise in intrauterine growth restriction Pediatr Res 42 514-9 PMID: 9380446 doi: 10.1203/00006450$199710000-00016$

36. Harrington B, Glazier J, D'Souza S and Sibley C (1999) System A amino acid transporter activity in human placental microvillous membrane vesicles in relation to various anthropometric measurements in appropriate and small for gestational age babies Pediatr Res 45 810-4 PMID: 10367770 doi: $10.1203 / 00006450-$ 199906000-00005

37. Speake PF, Glazier J, Ayuk PTY, Reade M, Sibley C and D'Souza S (2003) L-Arginine transport across the basal plasma membrane of the syncytiotrophoblast of the human placenta from normal and preeclamptic pregnancies J Clin Endocrinol Metab 88 4287-92 PMID: 12970300 doi: $10.1210 /$ jc. $2003-030067$

38. Cianciolo GJ, Copeland TD, Oroszlan S and Snyderman R (1985) Inhibition of Iymphocyte proliferation by a synthetic peptide homologous to retroviral envelope proteins Science 230 453-5 PMID: 2996136 doi: 10.1126/ science.2996136

39. Blaschitz A, Hutter H and Dohr G (2001) HLA Class I protein expression in the human placenta Early Pregnancy 5 67-9 PMID 11753519

40. Bennett WA, Lagoo-Deenadayalan S, Whitworth NS et al (1999) First-trimester human chorionic villi express both immunoregulatory and inflammatory cytokines: a role for interleukin-10 in regulating the cytokine network of pregnancy $A m \mathrm{~J}$ Reprod Immunol 41 70-8 PMID: 10097789

41. Ponferrada VG, Mauck BS and Wooley DP (2003) The envelope glycoprotein of human endogenous retrovirus HERV-W induces cellular resistance to spleen necrosis virus Arch Virol 148 659-75 PMID: 12664292 doi: $10.1007 / \mathrm{s} 00705-002-0960-\mathrm{x}$

42. Cheng $\mathrm{YH}$, Richardson $\mathrm{BD}$, Hubert MA and Handwerger $\mathrm{S}$ (2004) Isolation and Characterization of the Human Syncytin Gene Promoter Biol Reprod 70 694-701 PMID: 14613893 doi: $10.1095 /$ biolreprod.103.023473

43. Cheng $\mathrm{YH}$ and Handwerger S (2005) A placenta-specific enhancer of the human syncytin gene Biol Reprod 73 
500-9 PMID: 15888734 doi: 10.1095/biolreprod.105. $\underline{039941}$

44. Mortensen K, Lichtenberg J, Thomsen PD and Larsson LI (2004) Spontaneous fusion between cancer cells and endothelial cells Cell Mol Life Sci 61 2125-31 PMID: 15316661 doi: $10.1007 / \mathrm{s} 00018-004-4200-2$

45. Bjerregaard B, Holck S, Christensen IJ and Larsson LI (2006) Syncytin is involved in breast cancer-endothelial cell fusions Cell Mol Life Sci 63 1906-11 PMID: 16871371 doi: 10.1007/s00018-006-6201-9

46. Larsson JI, Bjerregaard B and Talts JF (2008) Cell fusions in mammals Histochem Cell Biol 129 551-61 PMID: $\underline{18351375}$ doi:10.1007/s00418-008-0411-1

47. Strick R, Ackermann S, Langbein $M$ et al (2007) Proliferation and cell-cell fusion of endometrial carcinoma are induced by the human endogenous retroviral syncytin-1 and regulated by TGF-beta $\mathrm{J} \mathrm{Mol}$ Med 85 23-38 PMID 17066266 10.1007/s00109-006-0104y

48. Uchida S, Shimada Y, Watanabe G, Li ZG, Hong T, Miyake M and Imamura M (1999) Motility-related protein (MRP. 1/CD9) and KAI1/ CD82 expression inversely correlate with lymph node metastasis in oesophageal squamous cell carcinoma Br J Cancer 79 1168-73 PMID: 10098753 doi:10.1038/sj.bjc.6690186
49. Hashida H, Takabayashi A, Tokuhara T et al (2002) Integrin alpha3 expression as a prognostic factor in colon cancer:association with MRP-1/CD9 and KAI1/CD82 Int J Cancer 97 518-25 PMID: 11802216 doi: 10.1002/ijc. 1625

50. Funakoshi T, Tachibana I, Hoshida $Y$ et al (2003) Expression of tetraspanins in human lung cancer cells:frequent downregulation of CD9 and its contribution to cell motility in small cell lung cancer Oncogene 22 674-87 PMID: 12569360 doi:10.1038/ sj.onc. 1206106

51. Matouskova M, Blazkova J, Pajer P, Pavlicek A and Hejnar $J$ (2006) CpG methylation suppresses transcriptional activity of human syncytin-1 in non-placental tissues Exp Cell Res 312 1011-20 PMID: 16427621 doi: 10.1016/ j.yexcr.2005.12.010

52. Menendez L, Benigno BB and McDonald JF (2004) L1 and HERV-W retrotransposons are hypomethylated in human ovarian carcinomas Mol Cancer 312 PMID: 15109395 doi: $10.1186 / 1476-4598-3-12$

53. Ogle BM, Cascalho M and Platt JL (2005) Biological implications of cell fusion Nat Rev Mol Cell Biol 6 56775 PMID: 15957005 doi: $10.1038 / \mathrm{nrm} 1678$

54. Larsson LI, Holck S and Christensen IJ (2007) Prognostic role of syncytin expression in breast cancer Hum Pathol 38 726-31 PMID 17306327 doi: 10.1016/j.humpath.2006. $\underline{10.018}$ 